TITLE:

\title{
Time-resolved detection of structural change in polyethylene films using mid-infrared laser pulses
}

\section{AUTHOR(S):}

Ageev, Eduard; Mizobata, Keisuke; Nakajima, Takashi; Zen, Heishun; Kii, Toshiteru; Ohgaki, Hideaki

\section{CITATION:}

Ageev, Eduard ...[et al]. Time-resolved detection of structural change in polyethylene films using mid-infrared laser pulses. Applied Physics Letters 2015, 107(4): 041904.

\section{ISSUE DATE:}

2015-07-29

\section{URL:}

http://hdl.handle.net/2433/202012

\section{RIGHT:}

(C) 2015 American Institute of Physics. This article may be downloaded for personal use only. Any other use requires prior permission of the author and the American Institute of Physics. 


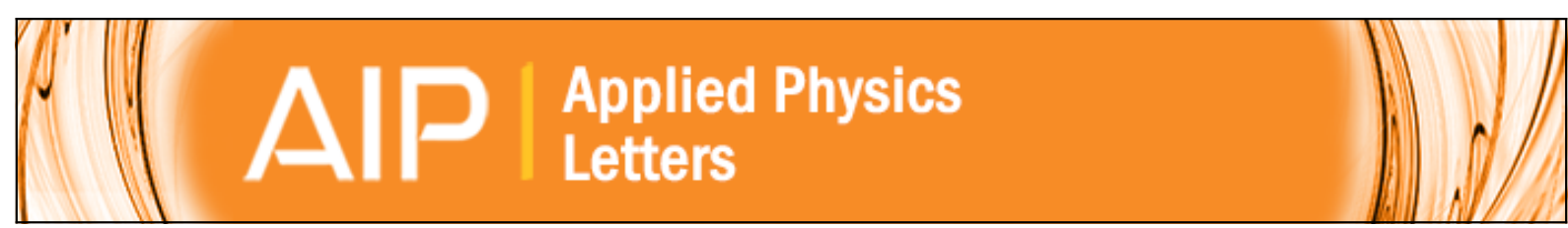

Time-resolved detection of structural change in polyethylene films using mid-infrared laser pulses

Eduard Ageev, Keisuke Mizobata, Takashi Nakajima, Heishun Zen, Toshiteru Kii, and Hideaki Ohgaki

Citation: Applied Physics Letters 107, 041904 (2015); doi: 10.1063/1.4927666

View online: http://dx.doi.org/10.1063/1.4927666

View Table of Contents: http://scitation.aip.org/content/aip/journal/apl/107/4?ver=pdfcov

Published by the AIP Publishing

\section{Articles you may be interested in}

Photolysis of $n$-butyl nitrite and isoamyl nitrite at $355 \mathrm{~nm}$ : A time-resolved Fourier transform infrared emission spectroscopy and ab initio study

J. Chem. Phys. 130, 174314 (2009); 10.1063/1.3129806

Infrared spectroscopic ellipsometry study of vinylidene fluoride (70\%)-trifluoroethylene (30\%) copolymer Langmuir-Blodgett films

J. Appl. Phys. 94, 195 (2003); 10.1063/1.1578697

Effect of ablation parameters on infrared pulsed laser deposition of poly(ethylene glycol) films

J. Appl. Phys. 91, 9809 (2002); 10.1063/1.1478136

Electronic linear energy transfer dependent molecular structural growth in polyethylene terephthalate J. Appl. Phys. 91, 4922 (2002); 10.1063/1.1462424

Time-resolved Raman spectroscopy of polytetrafluoroethylene under laser-driven shock compression Appl. Phys. Lett. 75, 947 (1999); 10.1063/1.124563

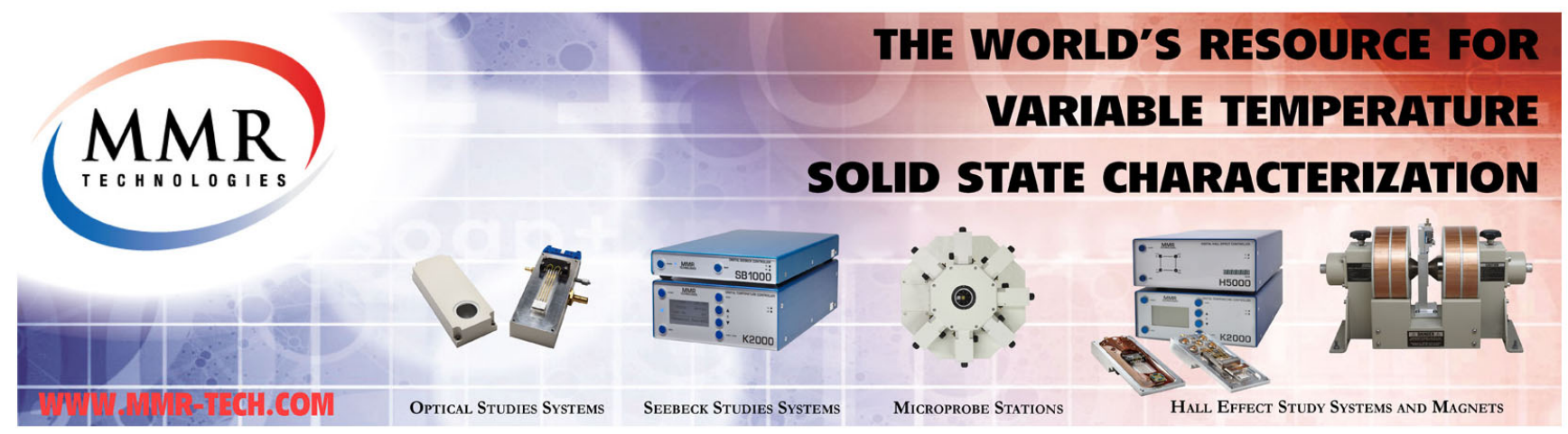




\title{
Time-resolved detection of structural change in polyethylene films using mid-infrared laser pulses
}

\author{
Eduard Ageev, Keisuke Mizobata, Takashi Nakajima, ${ }^{\text {a) }}$ Heishun Zen, Toshiteru Kii, \\ and Hideaki Ohgaki \\ Institute of Advanced Energy, Kyoto University, Gokasho, Uji, Kyoto 611-0011, Japan
}

(Received 3 June 2015; accepted 20 July 2015; published online 29 July 2015)

\begin{abstract}
Some of the vibrational modes of crystalline organic polymers are known to be sensitive to the structural change from the crystalline phase to the amorphous phase, and vice versa. Using a midinfrared (mid-IR) pulse from a free-electron laser as a probe, we demonstrate the time-resolved detection of structural change in crystalline polymer (polyethylene) films upon laser heating by a Q-switched Nd:YAG laser. Transmittance of the resonant mid-IR pulse almost instantaneously changes before and after the Nd:YAG laser pulse if its fluence is sufficient to induce the structural change in the film. The developed technique would be useful to study the time-dependent dynamics of the structural change in various materials. (C) 2015 AIP Publishing LLC.

[http://dx.doi.org/10.1063/1.4927666]
\end{abstract}

Various kinds of phase transitions have been attracting a lot of interests from the viewpoint of manifestation and control of the specific functions of materials. In the case of inorganic materials, magnetic phase transition ${ }^{1}$ and metalinsulator transitions ${ }^{2}$ are of great interest. On the other hand, in the case of organic materials, the structural phase change itself, which is of course accompanied by the change of the physical and chemical properties, is of great interest. Polymers are the commonly used organic materials for many purposes, and they are classified into two categories: amorphous polymers such as acryl and polystyrene and crystalline polymers such as polyethylene (PE) and polyethylene terephthalate (PET). We point out that the crystallinity of crystalline polymers is not $100 \%$, and they always contain the amorphous component to some extent. Although PE is the simplest and very commonly used crystalline polymer with only carbon and hydrogen atoms as constituent, there is no paper in the literature which reports the time-resolved study on its structural phase change.

To determine the structure of organic materials, there are many ways. Among others, mid-infrared (mid-IR) spectroscopy, such as FTIR and Raman spectroscopy, nuclear magnetic resonance, $\mathrm{x}$-ray diffraction, etc., are the well-established techniques. However, the available methods to detect the structural change in a time-resolved manner, which are more preferred to deepen the understanding of the dynamics, are rather limited. Spectroscopic techniques are convenient for insitu detection, but in many cases, the time-resolution of $\mathrm{FTIR}^{3,4}$ and Raman spectroscopy is not very high. One way to attain a certain time resolution is to use a pulsed laser and a conventional IR lamp for the pump and probe, respectively, together with a fast IR detector. ${ }^{5}$ A more straightforward way is to employ mid-IR pulses for the pump or probe pulses, or for both, and perform the pump-probe experiments. In recent years, mid-IR pulses based on a Ti:Sapphire laser system has been becoming available through the nonlinear frequency conversion techniques. ${ }^{6}$ Another way is to use mid-IR pulses

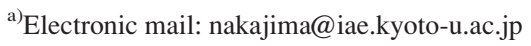

directly from a free-electron laser (FEL). Indeed, relaxation times of various vibrational modes of polymer films have been measured using mid-IR FELs with a picosecond time resolution. $^{7,8}$

In this paper, we report the time-resolved detection of structural change (crystalline $\rightarrow$ amorphous) in PE films using a mid-IR FEL developed at Kyoto University (KUFEL). ${ }^{9-11}$ To induce and detect the structural change in PE films, we employ two laser pulses: Q-switched Nd:YAG laser pulse to heat the PE film on an $\mathrm{NaCl}$ crystal and mid-IR FEL pulse to probe the structural change of PE. Note, however, that PE itself is practically transparent in the visible $\sim$ near-IR range, and the laser-heating with the Nd:YAG laser would not be efficient. Indeed, the brute force irradiation of the Nd:YAG laser pulse at high fluence onto the PE film results in the material damage before the sufficient heating takes place. To overcome this problem, the PE film is doped with absorptive dye which has large absorption around $1064 \mathrm{~nm} .^{12}$ There are various ways to detect the phase change: through the reflectivity change by the streak camera, ${ }^{13}$ transmission change of the probe pulse in the visible ${ }^{14}$ or $\mathrm{THz}$ range, ${ }^{1}$ or the change of the Raman signal. ${ }^{15}$ In this work, we measure the transmission change of the mid-IR probe pulse which is resonant with a structure-sensitive vibrational mode of PE. ${ }^{16}$ Currently, the time resolution is limited by the response of the mid-IR detector we employ, and it is a few hundreds of nanosecond time resolution. It can easily go down to a few nanoseconds only if the mid-IR detector is replaced by a faster one.

Dye-doped PE films we use in this study are fabricated in the following way. First, we dissolve a high-density PE pellet (Sigma-Aldrich, CAS: $9002-88-4$, mp:125-140 ${ }^{\circ} \mathrm{C}$ ) into the oDichlorobenzene solution (Wako special grade, CAS: 95-50-1) at $170^{\circ} \mathrm{C}$ on a hot plate for a few hours to obtain the solution with the PE concentration of $3 \mathrm{wt}$. \%. Then, we preheat a $\mathrm{NaCl}$ crystal $(25 \mathrm{~mm}$ diameter, $2 \mathrm{~mm}$ thickness) mounted on the spin-coater and a syringe tip (made of polypropylene) to $150^{\circ} \mathrm{C}$. The preheating of a $\mathrm{NaCl}$ crystal and a syringe tip is very important, since the use of a syringe tip at 
room temperature results in the immediate solidification of the PE solution in the tip. Recall that PE has very high chemical resistance to most of the organic solvents, and it is very difficult to dissolve PE. Similarly, a drop of the PE solution in a preheated syringe onto a $\mathrm{NaCl}$ crystal at room temperature results in the immediate solidification of the PE solution before spreading over the crystal surface. Just before the spincoating, we add the appropriate amount of IR165 dye (Exciton) into the PE solution at $150{ }^{\circ} \mathrm{C}$ to minimize the thermal damage of dye molecules. The dye concentration in the PE solution is carefully controlled to be $0.5 \mathrm{wt}$. \%. Using a surface profilometer, the thickness of the dye-doped PE film fabricated this way is found to be $2.0 \pm 0.4 \mu \mathrm{m}$.

Prior to the main experiment, we scan the wavelength of the FEL beam without introducing the Nd:YAG laser pulse and measure the transmission spectra through the PE film with two energy detectors (Gentec QE8SP-I-BL-BNC) of pyroelectric type for the reference and transmission signals to minimize the influence of the shot-to-shot fluctuation of FEL pulse energy. We repeat the similar measurement with a bare $\mathrm{NaCl}$ crystal to account for the transmission loss by the $\mathrm{NaCl}$ crystal itself. Thus, the transmission signals are converted to the transmittance. In Fig. 1, we show the transmittance of the dyedoped PE film at the film temperatures of 32 and $84{ }^{\circ} \mathrm{C}$ using a conventional heater. The position of the dip at $\sim 730 \mathrm{~cm}^{-1}$ looks slightly shifted by increasing the temperature. This is perhaps an experimental error when we scan the FEL wavelength. The FTIR spectrum of the same film at room temperature, $25^{\circ} \mathrm{C}$, is also shown for comparison. Because the FEL spectrum, which is initially about $25 \mathrm{~cm}^{-1}$, is appropriately narrowed through the monochromator (CVI DK240) with a slit width of $0.5 \mathrm{~mm}$, the FEL beam can clearly resolve the double-dip structure associated with the $\mathrm{C}-\mathrm{H}$ rocking mode (Fig. 6 of Ref. 16) of crystalline PE. Note that the PE film usually contains some amorphous component (Figs. 2 and 4 of Ref. 17), since the crystallinity of the PE film is not $100 \%$. Although $84{ }^{\circ} \mathrm{C}$ is still well below the melting point of $\mathrm{PE}$, we find the obvious increase of transmittance at the higher film temperature. Since the crystallinity of the PE film is known to decrease as the temperature increases, ${ }^{17}$ we can directly connect the increase of transmission at the resonant wavelength to the $\mathrm{C}-\mathrm{H}$ rocking mode of $\mathrm{PE}$ with the decrease of crystallinity.

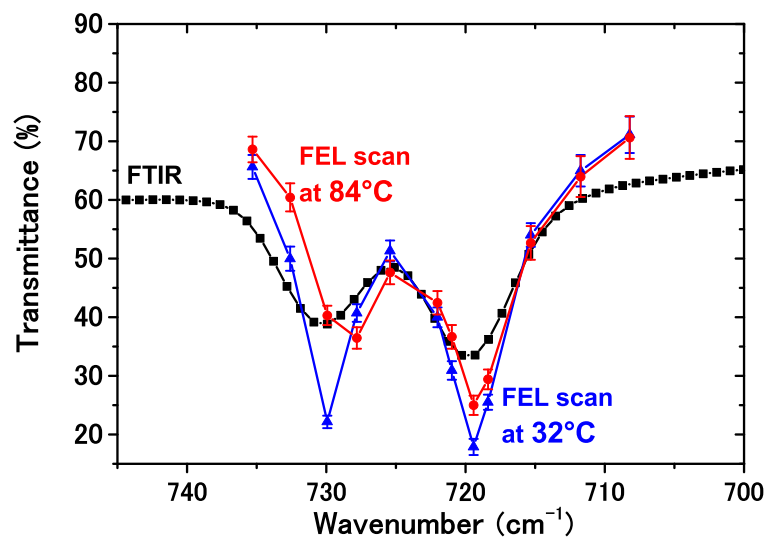

FIG. 1. Transmittance of the dye-doped PE film at 32 and $84{ }^{\circ} \mathrm{C}$ obtained by scanning the FEL wavelength. An FTIR spectrum of the same sample is also shown for comparison.
Therefore, the increase of transmission at the resonant wavelength is a clear indication of structural change from the crystalline phase to the amorphous phase. The above argument also implies that the structural change would not be very visible if the FEL beam is at the off-resonant wavelength.

The experimental setup for the time-resolved detection of structural change in dye-doped PE film is shown in Fig. 2. The FEL beam at $730 \mathrm{~cm}^{-1}(13.7 \mu \mathrm{m})$ with $\sim 10 \mathrm{~mJ}$ energy and $\sim 1.5 \mu$ s duration for the macropulse at the $1 \mathrm{~Hz}$ repetition rate goes through the monochromator (slit width $0.5 \mathrm{~mm}$ ) to narrow the FEL linewidth and then $2 \mathrm{~mm}$ aperture, as a result of which the FEL macropulse energy at the sample is less than several $\mu \mathrm{J}$. Each FEL macropulse contains a few thousands of micropulses with a sub-ps duration and 350 ps time interval, but our detectors do not have a time-response to resolve them. To minimize the influence of the shot-to-shot change of FEL pulse energy, we employ two photodetectors (Teledyne Judson Technologies J15D12M204-S01M-60) of mercury cadmium telluride (MCT) type for the reference and transmission signals. Since the MCT detectors have undesired sensitivity at the wavelength of the Nd:YAG laser (Rayture systems GAIA-III, $1064 \mathrm{~nm}, 5 \mathrm{~ns}$ pulse duration, max. $40 \mathrm{~mJ}$ pulse energy) we use to heat the PE film, long pass filters are placed in front of both detectors. To synchronize the Nd:YAG pulse and the FEL pulse, we use the radio-frequency (RF) pulse for electron acceleration of FEL as a master trigger for the delay generator (SRS DG645), through which the Q-switch of the Nd:YAG laser is triggered with an appropriate delay. The timing of the Nd:YAG laser pulse with respect to the RF pulse is adjusted so that the Nd:YAG laser pulse is turned on just after the peak of the FEL macropulse. There is tens of ns jitter between the RF pulse and the peak of the FEL pulse. This is due to the shot-to-shot difference of the amplification processes. This jitter, however, is negligible for the accuracy of the present experiment. The diameter of the Nd:YAG laser pulse is slightly shrunk with a convex lens to result in $4 \mathrm{~mm}$ diameter (defined for $1 / \mathrm{e}^{2}$ and measured by the beam profiler, Thorlabs BC106-VIS) at the sample, which is sufficiently larger than that of the FEL pulse.

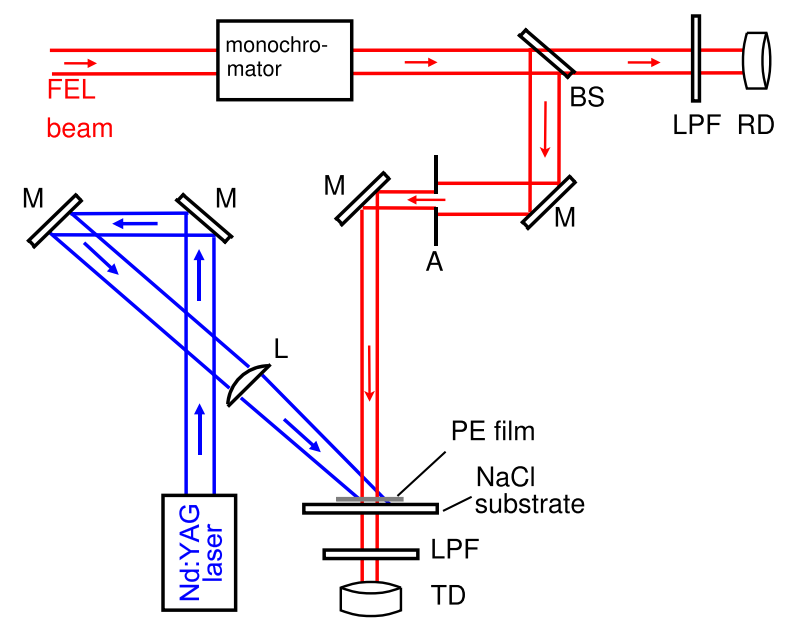

FIG. 2. Experimental setup. M, BS, L, A, and LPF stand for the gold mirror, $\mathrm{ZnSe}$ beam splitter, $\mathrm{f}=150 \mathrm{~mm}$ plano-convex $\mathrm{ZnSe}$ lens, $2 \mathrm{~mm}$ aperture, and long pass filter. RD and TD stand for the MCT detectors for the reference and transmission signals of FEL pulses, respectively. 


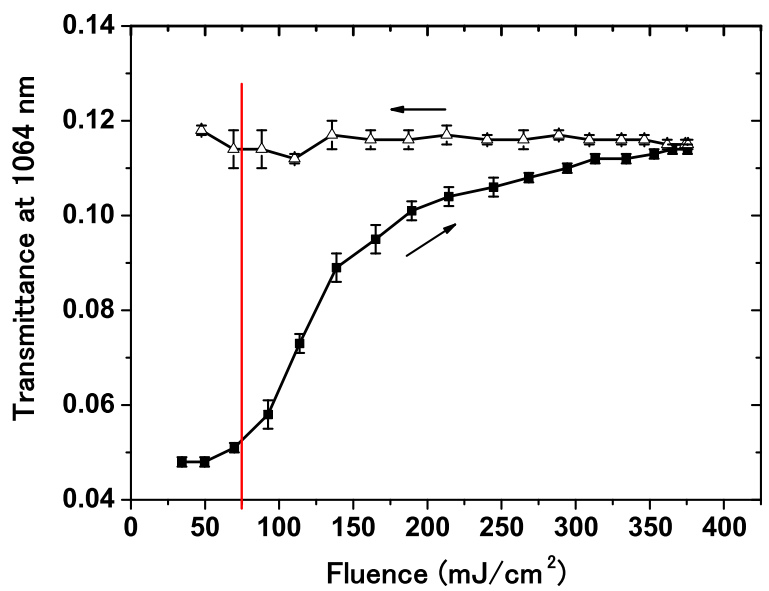

FIG. 3. Transmittance through the dye-doped PE film at $1064 \mathrm{~nm}$ as a function of Nd:YAG laser fluence. The laser fluence is first gradually increased from 30 to $390 \mathrm{~mJ} / \mathrm{cm}^{2}$ after the irradiation of 300 shots at $10 \mathrm{~Hz}$ for each fluence, and then gradually decreased back to $30 \mathrm{~mJ} / \mathrm{cm}^{2}$. The red vertical line at $75 \mathrm{~mJ} / \mathrm{cm}^{2}$ indicates the damage threshold of the dye-doped PE film. Note that the same position of the film is being irradiated during the entire measurements.

In Fig. 3, we show the transmittance at $1064 \mathrm{~nm}$ through the dye-doped PE film as a function of Nd:YAG laser fluence. We first increase the fluence from $30 \mathrm{~mJ} / \mathrm{cm}^{2}$ up to $390 \mathrm{~mJ} / \mathrm{cm}^{2}$ after the irradiation of 300 shots at $10 \mathrm{~Hz}$ at each fluence. At $75 \mathrm{~mJ} / \mathrm{cm}^{2}$, we notice the slight damage on the film surface by naked eyes, and we define this fluence as damage threshold. We further increase the fluence up to 390 $\mathrm{mJ} / \mathrm{cm}^{2}$. Increase of transmittance at the higher fluence implies that more dye molecules are destroyed by the irradiation of more intense laser. After the fluence of $390 \mathrm{~mJ} / \mathrm{cm}^{2}$ is reached, we gradually decrease the fluence back to $30 \mathrm{~mJ} / \mathrm{cm}^{2}$. During the decrease of fluence, the transmittance at $1064 \mathrm{~nm}$ remains to be the same, as expected.

Now, we tune the FEL pulse to be resonant with the absorption line of PE film at $730 \mathrm{~cm}^{-1}$ and measure its transmission through the PE film with the Nd:YAG laser on at the fluence of $77 \mathrm{~mJ} / \mathrm{cm}^{2}$ to find that there is no transmission change of the FEL pulse before and after the Nd:YAG laser pulse (Fig. 4(a)). It is not until $390 \mathrm{~mJ} / \mathrm{cm}^{2}$ that we observe the clear transmission change before and after the Nd:YAG laser pulse (Fig. 4(b)). In order to ensure that this transmission change is indeed due to the structural change of the dyedoped PE film, we carry out a similar experiment at the same Nd:YAG laser fluence but with the off-resonant FEL wavelength of $712 \mathrm{~cm}^{-1}$ (Fig. 4(c)). From the comparison of Figs. 4(a)-4(c), we can confidently conclude that the transmission increase of the resonant FEL pulse upon irradiation of the Nd:YAG laser pulse (Fig. 4(b)) with sufficient fluence must be due to the structural change (crystalline $\rightarrow$ amorphous) of the PE film upon melting by laser heating. The slight modulations in the FEL signals of Fig. 4 are due to the noise from the RF source for electron acceleration of FEL, which is different from shot-to-shot, and hence, it cannot be subtracted when we plot the transmission change. The increase of transmission change in Fig. 4(b) is in the time scale of $\sim \mu \mathrm{s}$, which is much longer than the time for the pressure-induced phase change in polytetrafluoroethylene (PTFE). ${ }^{15}$ By noticing that the local temperatures are different at different film

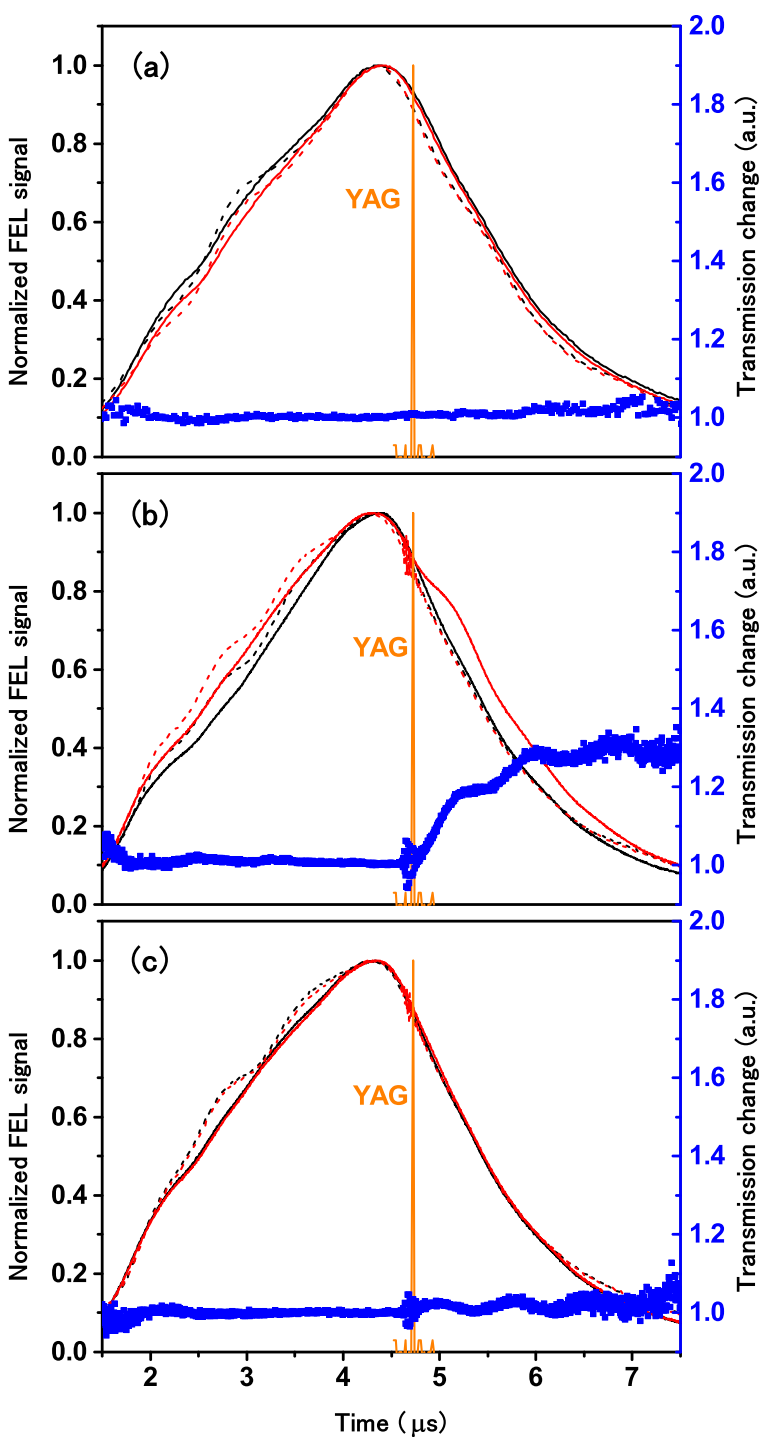

FIG. 4. Transmission change of the FEL pulse through the dye-doped PE film before and after the Nd:YAG laser pulse. The employed FEL wavelength and Nd:YAG laser fluence are (a) $730 \mathrm{~cm}^{-1}$ and $77 \mathrm{~mJ} / \mathrm{cm}^{2}$, (b) $730 \mathrm{~cm}^{-1}$ and $390 \mathrm{~mJ} / \mathrm{cm}^{2}$, and (c) $712 \mathrm{~cm}^{-1}$ and $390 \mathrm{~mJ} / \mathrm{cm}^{2}$, respectively. In each panel, the transmission and reference signals with (without) $\mathrm{Nd}$ :YAG laser pulses are shown by the solid (dashed) red and black curves, respectively, while the transmission change, calculated by the transmission signals divided by the reference signals, is shown by the blue curve. Temporal position of the Nd:YAG laser pulse is shown by the sharp orange line around $4.7 \mu \mathrm{s}$ in each panel. All results are obtained after averaging the data over 50 shots.

depths in our case because neither Nd:YAG laser fluence nor distribution of dye molecules are uniform (see Fig. 2), we expect that the apparent response time for the transmission change would be much longer than the intrinsic response time for the structural change of a single polymer. This explains why the time scale for the phase change we find in Fig. 4 (b) is $\sim \mu$ s. In the time range of several $\mu$ s, we only observe the transmission increase, and do not observe the decrease back to the initial value. This implies that the time needed for the film to cool down is much longer than this time scale.

Before closing the paper, we make a rough estimation of the film temperature increase upon irradiation of Nd:YAG laser pulses. This is not an easy task, since the dye 
concentration is not uniform along the laser propagation axis. Recall that the Nd:YAG laser pulse at high fluence destroys some of the dye molecules as we have seen in Fig. 3. Qualitatively, however, we can say, that, at the position deeper in the film, more dye molecules survive the irradiation of intense Nd:YAG laser pulse. From the experimental fact that we can see the obvious transmission change of the resonant FEL pulse at the Nd:YAG laser fluence of $390 \mathrm{~mJ} / \mathrm{cm}^{2}$ and not below this value, we consider that the temperature at the deepest part of the film can go beyond the melting point around this fluence. Assuming that none of the dye molecules are destroyed and its concentration is nearly uniform at the film depth of $1.9-2.0 \mu \mathrm{m}$, which means that the Beer's law is valid at this film depth, we can calculate the absorbed pulse energy to be about $1.3 \%$ with the aid of Fig. 3. Then, using the formula of $F A=\rho h c(\Delta T+L / c)$ where $F$ is Nd:YAG laser fluence, $A$ is absorptance, and $\rho, h, c, L$ are the density $\left(0.952 \mathrm{~g} / \mathrm{cm}^{3}\right)$, thickness $(\sim 2 \mu \mathrm{m})$, heat capacity $\left(2.3 \mathrm{~J} / \mathrm{g}{ }^{\circ} \mathrm{C}\right)$, and latent heat $(286.2 \mathrm{~J} / \mathrm{g})$ of PE with $\Delta T$ being the temperature increase, we obtain $\Delta T=114^{\circ} \mathrm{C}$ at the deepest part $(1.9-2.0 \mu \mathrm{m})$ of the PE film. This agrees well with the minimum temperature increase, $110^{\circ} \mathrm{C}$, required to melt the PE film.

In conclusion, we have demonstrated the time-resolved detection of structural change in crystalline polymer (polyethylene) films upon laser heating. When we tune the mid-IR probe pulse to the structure-sensitive vibrational mode of polyethylene, transmission of the probe pulse clearly changes before and after the laser heating pulse if the fluence of the heating pulse is sufficient. The experimental technique we have developed in this work would be useful to study the time-dependent dynamics of the structural change in various polymer materials. For the more detailed study of the dynamics, it is essential to fabricate a thinner ( $<\mu$ m thickness) polymer film with better quality so that we can see the structural change without damaging the dye molecules and carry out more refined experiments to clarify not only the heating but also the cooling processes to understand the re-crystallization dynamics, which is underway in our group.

We acknowledge Professor Takashi Morii for kindly letting us use their FTIR. This work was supported by a Grant-in-Aid for Scientific Research from the Ministry of Education, Culture, Sports, Science and Technology of Japan.

${ }^{1}$ M. K. Liu, B. Pardo, J. Zhang, M. M. Qazilbash, S. J. Yun, Z. Fei, J.-H. Shin, H.-T. Kim, D. N. Basov, and R. D. Averitt, Phys. Rev. Lett. 107, 066403 (2011).

${ }^{2}$ T. Z. Ward, X. G. Zhang, L. F. Yin, X. Q. Zhang, M. Liu, P. C. Snijders, S. Jesse, E. W. Plummer, Z. H. Cheng, E. Dagotto, and J. Shen, Phys. Rev. Lett. 102, 087201 (2009).

${ }^{3}$ W. Uhmann, A. Becker, C. Taran, and F. Siebert, Appl. Spectrosc. 45, 390 (1991).

${ }^{4}$ S. V. Shilov, H. Skupin, F. Kremer, T. Wittig, and R. Zentel, Phys. Rev. Lett. 79, 1686 (1997).

${ }^{5}$ S. Shen, X. Wang, T. Chen, Z. Feng, and C. Li, J. Phys. Chem. C 118, 12661 (2014).

${ }^{6}$ R. A. Kaindl, M. Wurm, K. Reimann, P. Hamm, A. M. Weiner, and M. Woerner, J. Opt. Soc. Am. B 17, 2086 (2000).

${ }^{7}$ D. Zimdars, A. Tokmakoff, S. Chen, S. R. Greenfield, M. D. Fayer, T. I. Smith, and H. A. Schwettman, Phys. Rev. Lett. 70, 2718 (1993).

${ }^{8}$ J. R. Wells, D. G. Lidzey, P. J. Phillips, D. A. Carder, and A. M. Foz, Appl. Phys. Lett. 85, 3080 (2004).

${ }^{9}$ H. Ohgaki, T. Kii, K. Masuda, H. Zen, S. Sasaki, T. Shiiyama, R. Kinjo, K. Yoshikawa, and T. Yamazaki, Jpn. J. Appl. Phys., Part 1 47, 8091 (2008).

${ }^{10}$ X. Wang, T. Nakajima, H. Zen, T. Kii, and H. Ohgaki, Opt. Lett. 37, 5148 (2012).

${ }^{11}$ X. Wang, T. Nakajima, H. Zen, T. Kii, and H. Ohgaki, Appl. Phys. Lett. 103, 191105 (2013).

${ }^{12}$ X. Wen, W. A. Tolbert, and D. D. Dlott, Chem. Phys. Lett. 192, 315 (1992).

${ }^{13}$ J. Bonse, S. M. Wigging, J. Solis, and T. Lippert, Appl. Surf. Sci. 247, 440 (2005).

${ }^{14}$ Y. Saito, Y. Sutou, and J. Koike, Appl. Phys. Lett. 102, 051910 (2013).

${ }^{15}$ H. Nagao, A. Matsuda, K. G. Nakamura, and K. Kondo, Appl. Phys. Lett. 83, 249 (2003).

${ }^{16}$ R. G. Snyder, M. Maroncell, H. L. Strauss, and V. M. Hallmark, J. Phys. Chem. 90, 5623 (1986).

${ }^{17}$ H. Hagemann, R. G. Snyder, A. J. Peacock, and L. Mandelkern, Macromolecules 22, 3600 (1989). 